\title{
Scheduler-dependent inter-cell interference and its impact on LTE uplink performance at flow level
}

\author{
D.C. Dimitrova ${ }^{1 \star}$, G. Heijenk ${ }^{1}$, J.L. van den Berg ${ }^{1,2}$, and S. Yankov ${ }^{3}$ \\ 1 University of Twente, Enschede, The Netherlands \\ 2 TNO ICT, Delft, The Netherlands \\ 3 Technical University Sofia, Bulgaria
}

\begin{abstract}
The Long Term Evolution (LTE) cellular technology is expected to extend the capacity and improve the performance of current 3G cellular networks. Among the key mechanisms in LTE responsible for traffic management is the packet scheduler, which handles the allocation of resources to active flows in both the frequency and time dimension. This paper investigates for various scheduling scheme how they affect the inter-cell interference characteristics and how the interference in turn affects the user's performance. A special focus in the analysis is on the impact of flow-level dynamics resulting from the random user behaviour. For this we use a hybrid analytical/simulation approach which enables fast evaluation of flow-level performance measures. Most interestingly, our findings show that the scheduling policy significantly affects the inter-cell interference pattern but that the scheduler specific pattern has little impact on the flow-level performance.
\end{abstract}

\section{Introduction}

A key feature of packet scheduling in LTE networks is the possibility to schedule not only in time (as in UMTS/EUL) but also in frequency. The latter is enabled by the orthogonality between sub-carriers in SC-FDMA (Single Carrier Frequency Division Multiple Access) - the radio access technology chosen in LTE uplink. The smallest scheduling unit is termed a resource block (RB), see [6], and is the intersection between the frequency and time domain scheduling units. Due to the organisation of the spectrum into resource blocks and the various allocation approaches of these over the users, each resource block may experience different inter-cell interference. Hence, we can observe multiple inter-cell interference processes each of which behaves as a stochastic process.

Inter-cell interference for LTE networks has been widely researched. Interestingly most studies concentrate on performance evaluation of packet scheduling mechanisms with interference mitigation either based on inter-cell interference

\footnotetext{
* Corresponding author current affiliation: dimitrova@iam.unibe.ch, Neubruckstrasse 10, CH 3012 Bern, Switserland.
} 
coordination, see $[7,10,12]$, or based on pre-existing knowledge of the interference, see $[2,9,11]$. Still, they do not provide insights on the inter-cell interference process itself. We argue however that such insights are valuable for the design of an optimal allocation strategy. Therefore, in this study we take a step back and build an understanding of the mutual interaction between scheduling and interference. This is done in the context of LTE uplink with special focus on flow-level behaviour.

Studies that are closely related to our research are [5] and [8]. In [5] two allocation schemes for LTE downlink with distinct frequency allocation approaches are compared taking into account flow-level dynamics. Despite being very insightful the research does not provide information on how the inter-cell interference pattern is affected by the type of allocation. It also does not discuss flow-level performance in terms of throughput (only SINR is evaluated). The authors of [8] provide a valuable study on basic scheduling schemes and their impact on inter-cell interference. Unfortunately, the study considers only averaged metrics to describe the flow-level performance. The research inspired us to study the inter-cell interference process in an LTE uplink in more detail, including both scheduler's specifics and user behaviour.

The novelty of our research lays in two aspects. On the one hand, in the context of LTE uplink, we determine the dependency of the inter-cell interference on the scheduling strategy and, in particular, on the various ways to order users for service. On the other hand, user performance is evaluated at flow level taking into account the continuously changing number of ongoing flows in a system.

Previously, see [4], we showed that flow-level evaluation reveals important performance trends in the context of UMTS/EUL. We expect this general observation to hold for an LTE network as well; however we expect different outcomes. On the one hand, additional complexity is introduced by the new freedom to schedule in the frequency domain. On the other hand, due to the orthogonality of sub-carriers in LTE intra-cell interference is no more an issue. In order to investigate that we applied a significantly extended version of the hybrid analysis approach developed in [4]. The basic separation between packet level (to capture specifics of the scheduler and the radio environment) and flow level (to model the random user behaviour) is preserved. However, we introduce additional means at packet level to capture the changes in inter-cell interference in both time and frequency. Further, at flow level the generation and completion of finite-sized flows is modelled by Markov chains from whose steady-state distribution performance measures, e.g. mean flow transfer times, were derived. Detailed discussion of the current research can be found in [3].

The paper continues with Section 2, which elaborates on the investigated scheduling aspects. The system model is presented in Section 3, while Section 4 discusses the applied analysis approach. The discussion on numerical results is split over Section 5 (a study on the inter-cell interference process) and Section 6 (discussion of performance results). Finally, we conclude with Section 7. 


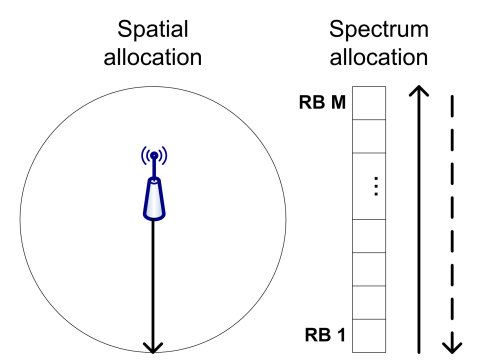

(a) CE policies

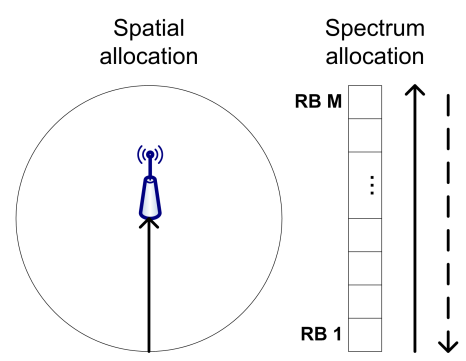

(b) EC policies

Fig. 1. User ordering policies for scheduling in LTE uplink. (a) CE-begin (solid arrow) and CE-end (dashed arrow) and (b) EC-begin (solid arrow) and EC-end (dashed arrow).

\section{$2 \quad$ Interference-aware scheduling}

In LTE we have the freedom to schedule in both time and frequency domain with the smallest scheduling unit being a resource block (RB). In particular, we denote by a resource block ${ }^{4}$ a unit of bandwidth of $180 \mathrm{kHz}$ and duration of $1 \mathrm{~ms}$. If we denote by $M$ the total number of RBs within the available system bandwidth during a single TTI, the scheduler can distribute at most $M$ resource blocks over the active users. The total bandwidth that can be allocated to a single MS depends on the resource availability, the radio link quality and the terminal's transmit power budget. Although intra-cell interference is hardly an issue due to the sub-carrier orthogonality in SC-OFDM, situations are still possible when transmissions of users (allocated the same resource blocks) in neighbouring cells interfere with each other.

The scheduling scheme studied in our analysis is a resource fair scheduling scheme, which assigns equal resource shares to all active users, independently of their respective channel conditions. We consider the case of single resource block allocation and thus, given a total of $M$ RBs in the total LTE uplink bandwidth, there are at most $M$ users that can be served in one TTI. As a consequence there are $M$ inter-cell interference processes - one for each RB.

An aspect of the scheduling is the ordering policy. The ordering policy dictates in which sequence users are selected for service and in which order the RBs are allocated to the selected users. We base the user selection on the location of the user in the cell. This spatial aspect is important since users at different locations generate different inter-cell interference values at the base station of a neighbour cell. Users close to the base station tend to generate similar levels. On the contrary, the interference levels from users located towards the cell edge vary significantly depending on their relative position to a neighbour cell. A user close to the neighbour cell generates high interference while a user on the opposite end (farthest from the neighbour cell) generates low interference levels.

\footnotetext{
${ }^{4}$ In standards a resource block refers to the intersection of $180 \mathrm{kHz}$ and $0.5 \mathrm{~ms}$.
} 


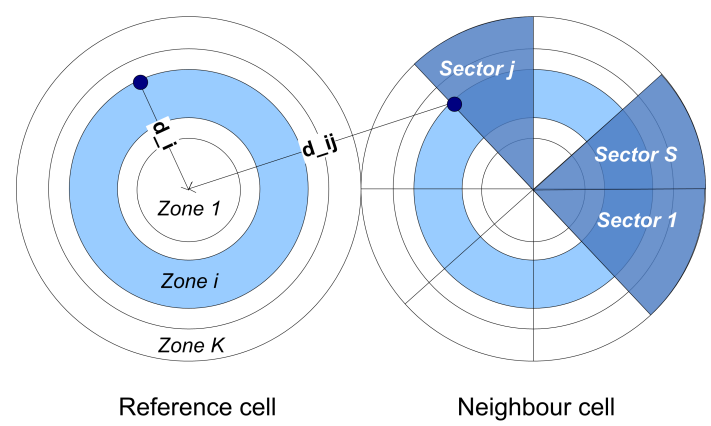

Fig. 2. System model - reference cell and neighbour cell.

We have defined five distinct ordering policies accounting for both the spatial and frequency aspect of ordering. The first policy, which is also our reference case, is termed random and as the name suggests the selection of users and the allocation of RBs is based on a random principle, i.e. independently of the user's location. The other four policies have a more 'structured' approach. The $C E$-begin policy starts allocating RBs from the low end of the used spectrum to users closest to the base station and proceeds with farther located ones, see Figure 1(a) solid arrow. The CE-end policy, presented also in Figure 1(a), but dashed arrow, adopts the same spatial order but starts assigning first RBs from the high end of the spectrum. The EC-begin (see Figure 1(b), solid arrow) and $E C$-end (see Figure 1(b), dashed arrow) policies both start serving first the users located farthest from the base station; the former starts assigning RBs from the beginning of the spectrum while the latter starts from the high end of the spectrum. Note that the CE-begin and EC-end policies in fact differ since not at all times the total spectrum is occupied.

\section{Model}

We consider a two cell system - one cell (neighbour cell NC), where the interference originates, and one cell (reference cell RC), where its effect on user performance is observed. Such model, see Figure 2, suffices to observe the impact of the scheduler on the inter-cell interference pattern. The reference cell is divided in $K$ zones of equal area which allows us to distinguish in user's performance depending on its location in the cell, i.e. distance to the base station. Each zone is characterised by a distance $d_{i}$ to the base station, $i=1, \ldots, K$, measured from the outer edge of the zone. Additionally, to model the effect of user location on interference, the neighbour cell is divided in $S$ sectors of equal area. The intersection of a zone and a sector is termed a segment, which is characterised by a distance $d_{i}$ to the base station of the NC and a distance $d_{i j}$ to the base station of the $\mathrm{RC}, j=1, \ldots, S$. The number of users in a cell and their distribution is given by the system state $\underline{n}$. Given the chosen cell model the RC is characterised by 
$\underline{n} \equiv\left(n_{1}, n_{2} \ldots n_{K}\right)$ and the $\mathrm{NC}$ by $\underline{n} \equiv\left(n_{11}, n_{12}, \ldots, n_{1 S}, n_{21}, n_{22}, \ldots, n_{K S}\right)$. We assume that the $\mathrm{NC}$ depends independently and is does not experience interference from the RC.

Further, users are uniformly distributed over the cell(s) and initiate new flow transfers according to a Poisson arrival process with rate $\lambda$. Flow size is exponentially distributed with mean $F$. Given the equal size of zones and sectors we can define arrival rate per zone (for the RC) $\lambda_{i}=\lambda / K$ and arrival rate per segment (for the NC) $\lambda_{i j}=\lambda /(K S)$. The number of active users is limited to the maximum number of resource blocks $M$ that fit in the LTE uplink bandwidth. This is a reasonable assumption, which significantly eases analysis without biasing results $^{5}$. Note that the assumption holds only for the scheduling schemes that we study; for other schemes it might not be applicable. Users apply a maximum transmit power $P_{\max }^{t x}$ unless lower level suffice. The signal received at the base station is affected by path loss and inter-cell interference. We apply the COST 231 Hata propagation model, see [6], giving the path loss in logarithmic scale as:

$$
L\left(d_{i}\right)=L_{f i x}+10 a \log _{10}\left(d_{i}\right),
$$

where $L_{f i x}$ is a system parameter and $a$ is the path loss exponent.

\section{Analysis}

The analysis of the two-cell model described in Section 3 needs to account for three aspects - (i) the inter-cell interference from the neighbour cell, (ii) modelling the inter-cell interference in the reference cell and (iii) user performance at flow level in the reference cell. The proposed analysis approach to tackle these issues is described in Section 4.2. Before that Section 4.1 discusses the user behaviour within a single cell ( $\mathrm{NC}$ or $\mathrm{RC}$ ), which is the basis for the two-cell system modelling.

\subsection{Individual cell analysis}

Since a user applies a $P_{\max }^{t x}$ even if a lower power would be sufficient to achieve the highest possible data rate, we are looking at a worst-case interference scenario. Accounting for thermal noise $N$ and inter-cell interference $I_{o c}$, the signal-tointerference-plus-noise ratio (SINR) is given as:

$$
S I N R_{i}=\frac{P_{i}^{r x}}{N+I_{o c}},
$$

where $i$ indicates the zone number, $i=1, \ldots, K$, and the received power $P_{i}^{r x}$ is calculated according to $P_{i}^{r x}=P_{i}^{t x} / L\left(d_{i}\right)$. Note that the $I_{o c}$ component depends on whether the reference or the neighbour cell is modelled. In the calculation of

\footnotetext{
${ }^{5}$ Our observations show that situations with more than $M$ users form $10 \%$ of all observations for very high load but are below $1 \%$ for medium and low loads.
} 
the $S I N R$ we have considered an upper and lower bound set by the technically feasible modulation and coding schemes (MCSs) and the minimum required level for successful reception respectively.

The data rate realised by a user (from zone $i$ ) when it is scheduled is what we term instantaneous rate $r_{i}$. It is determined by the SINR as derived above, the possible MCSs and the receiver characteristics related to that MCS. In our analysis we use the Shannon formula modified with a parameter $\sigma$ to represent the limitations of implementation, see Annex A in [1]. Hence, for the instantaneous rate we can write:

$$
r_{i}=(m \cdot 180 \mathrm{kHz}) \sigma \log _{2}\left(1+S I N R_{i}\right),
$$

where $180 \mathrm{kHz}$ is the bandwidth of a single RB and $m$ is the number of RBs allocated to a user (in our case one). Note that both $S I N R_{i}$ and $r_{i}$ are calculated over the same RB allocation.

Flow dynamics, i.e. the changing number of active users over time, are incorporated in our analysis by means of a continuous time Markov chain. A state in the model corresponds to the state $\underline{n}$ and the transitions rates are determined as follows: the forward transition rates are taken from the Poisson arrival rate $\lambda$; the backward transition rates are derived from the instantaneous rate $r_{i}$ taking into account the mean flow size $F$ resulting in $r_{i} / F$. The form of the Markov chain of a single cell depends on the modelling, e.g. the Markov chain of the RC has $K$ dimensions corresponding to the $K$ zones while The Markov chain of the neighbour cell is $K \times S$ dimensional (due to the division in segments). From the steady-state distribution of the Markov chains performance metrics such as mean flow transfer times can be derived. Generally, the steady-state distribution can be found either analytically or, when necessary, by simulation. More elaborate description of the model can be found in [3].

\subsection{Two-cell system performance}

Applying the analysis from Section 4.1 to both the $\mathrm{RC}$ and $\mathrm{NC}$ we are able to evaluate the performance of the two-cell model, see Section 3. The evaluation goes through three phases.

Phase 1 In phase one we study the inter-cell interference generated by the neighbour cell towards the reference cell as a stochastic process. We are particularly interested in the observed interference levels and their probability distribution, reflected by the cumulative distribution function and the entire interference process. Generally, each segment in the NC corresponds with a different interference level. This since the inter-cell interference is in fact the received power at the base station of the reference cell from a user in the neighbour cell. Hence, the inter-cell interference from a segment $i j$ can be derived as:

$$
I_{o c}^{i j}=\frac{P_{\max }^{t x}}{L\left(d_{i j}\right)}, i=1, \ldots, K, j=1, \ldots, S .
$$

The probability distribution is determined by how often each particular value exhibits for a specific observation period. Hence, it depends on the traffic load 
and on the chosen scheduling (and ordering) policy. By applying the single-cell analysis for the neighbour cell we can derive the probability of each $I_{o c}$ level.

Phase 2 At this phase we account for the inter-cell interference at the base station of the reference cell. This is easily done due to the assumption that there are at most $M$ users in the system. As result, given a state $\underline{n}$, a user is always assigned the same $\mathrm{RB}$, i.e. in the $\mathrm{RC}$ each user always experiences the same inter-cell interference. The exact interference value per RB per state and the duration for which it exhibited (how long the NC stayed in each state) are taken from a trace file generated at phase one.

Phase 3 The third and last phase of the evaluation is determining the user performance in the reference cell at the flow level. For this again the singlecell analysis is used but applied to the RC. In building the Markov model we dynamically account for the inter-cell interference measured as described in phase two. By simulating the Markov chain of the reference cell we derive its steadystate distribution and subsequently the mean flow transfer time for each zone.

\section{$5 \quad$ Numerical results - inter-cell interference}

In this section we present a discussion on the inter-cell interference levels and probability distribution for each of the ordering policies described in Section 2. Before that we give the applied parameter settings.

\subsection{Parameter Settings}

An LTE system with $10 \mathrm{MHz}$ bandwidth is studied, which, given that a RB has $180 \mathrm{kHz}$ bandwidth, results in $50 \mathrm{RBs}$ available per TTI (including control overhead). Cell radius is set to $1 \mathrm{~km}$. Cell division in ten zones, i.e. $K=10$, and ten sectors (for NC), i.e. $S=10$, is applied ${ }^{6}$. For the path loss we have used $P L_{f i x}=141.6 \mathrm{~dB}$ considering height of the mobile station $1.5 \mathrm{~m}$, height of the eNodeB antenna $30 \mathrm{~m}$ and system frequency $2.6 \mathrm{GHz}$; and a path loss exponent of $a=3.53$. The thermal noise per sub-carrier $(180 \mathrm{kHz})$ is $-121.45 \mathrm{dBm}$ and with a noise figure of $5 \mathrm{~dB}$ the effective noise level per resource block is $N=$ $-116.45 \mathrm{dBm}$. The attenuation of implementation $\sigma$ is taken at 0.4 flows/sec, see [1]. Mobile stations have a maximum transmit power $P_{\max }^{t x}=0.2$ Watt. The lower bound on the $S I N R$ is $-10 \mathrm{~dB}$ while the upper bound on performance is determined by a 16QAM modulation, which corresponds to a $S I N R$ of $15 \mathrm{~dB}$. The average file size $F$ is $1 \mathrm{Mbit}$ and the rate $\lambda$ at which users become active changes depending on the discussed scenario.

\subsection{Inter-cell interference process}

We start with a discussion on the inter-cell interference values that a user in the neighbour cell generates at the base station of the reference cell. The results

\footnotetext{
${ }^{6}$ Finer division increases the differentiation ability in interference values but also the complexity of the model and simulation time. Tests with finer granularity did not show a significant change in the results.
} 


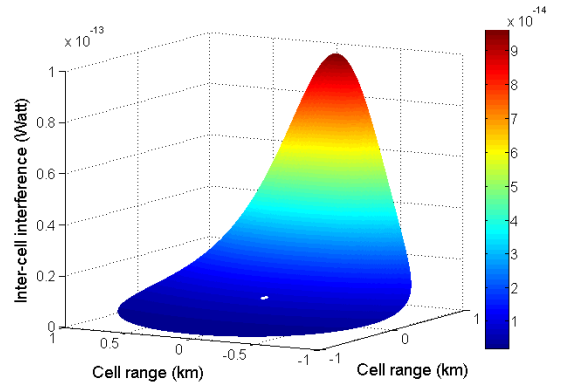

Fig. 3. Inter-cell interference levels.

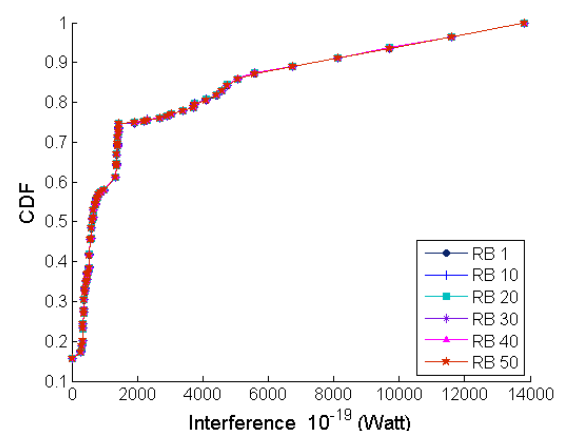

Fig. 4. CDF of inter-cell interference levels for the random ordering policy.

are shown in Figure 3. The $\mathrm{x}$ and $\mathrm{y}$ axes specify the location of the user in the $\mathrm{NC}$ while the $\mathrm{z}$ axis indicates the values of the inter-cell interference. The base station of the RC in located at coordinates $(2,0)$. Users close to the RC generate the highest interference levels as visible in the sharply increasing values for locations close to the RC, i.e. coordinates $(1,-1: 1)$. Furthermore, users from the cell centre generate interference levels close in values, i.e. with low variance, while the interference from users towards the cell edge can differ significantly, i.e. has high variance.

A cumulative distribution function (CDF), although hiding the chronological development of the inter-cell interference, can provide valuable insights. Note that for each ordering scheme there are 50 inter-cell interference processes, one for each RB, see Section 2. We present the CDFs of the processes observed at RBs numbers 1, 10, 20, 30, 40 and 50. In all figures the $\mathrm{x}$-axis indicates the observed inter-cell interference levels, while the y-axes plots the CDF function for each of the selected RBs. The arrival rate $\lambda$ per cell is 4 flows/sec, which is close to the maximum of what the system can support, i.e. $\lambda=4.8$ flows $/ \mathrm{sec}$ with zero inter-cell interference.

Figure 4 presents the CDF curves observed in the case of a random ordering policy. As we can see the plots coincide, which can be explained by the random manner in which users are selected for service and are assigned RBs. For example, while at one time instant on RB1 a user from the centre can be served at the next instant a user from the cell edge can be chosen. Hence, each possible inter-cell interference level has equal chance to exhibit at any RB. The steep initial part of the curves is determined by the many segments with similar low $I_{o c}$ levels, see Figure 3.

Let us now consider the policies with strictly defined order of service shown in Figure 5. We notice that the graphs of the CE-begin and the CE-end policies are mirror images, as well as the graphs of the EC-begin and EC-end policies. This is the result of the same spatial ordering of users that each set of policies applies but starting from the opposite ends of the spectrum. 


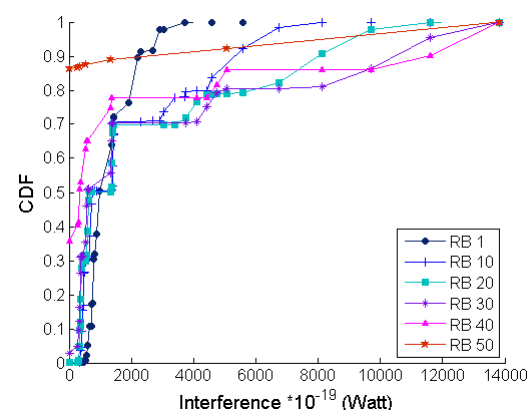

(a) CE-begin

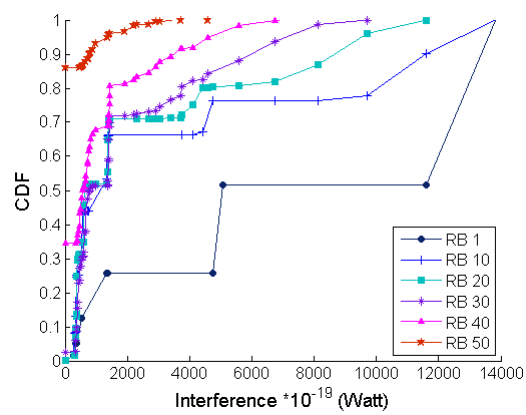

(c) EC-begin

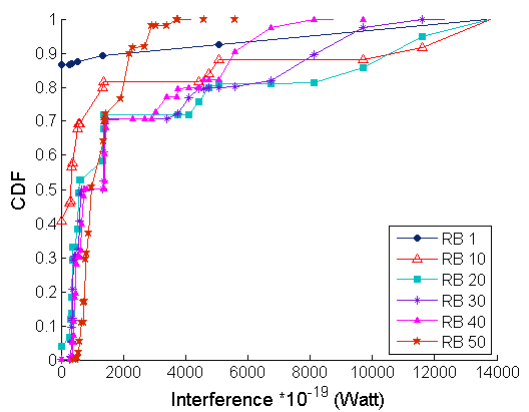

(b) CE-end

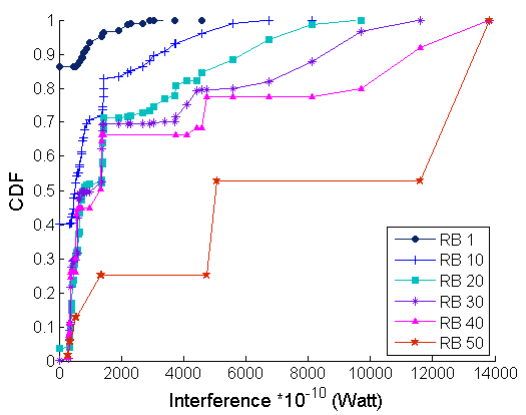

(d) EC-end

Fig. 5. CDFs of the inter-cell interference for the four pre-set ordering policies.

Focusing on the CE-begin policy, presented in Figure 5(a), we observe that the CDF curves for different RBs differ significantly. As we move towards the end of the spectrum, initially (from RB1 to RB30) the probability that high inter-cell interference values occur increases. Subsequently however low (including zero) interference values are more often observed, i.e. have higher probability towards the end of the allocated spectrum (e.g. for RB40 and RB50). This behaviour is the combined result of two factors. On the one hand, with the particular spatial ordering (centre to edge), edge users receive RBs towards the end of the spectrum. These users, when located close to the reference cell, have much higher interference levels than centre users, which explains the initial increase in the probability of high interference levels. On the other hand, RBs at the high end of the spectrum are occasionally left unused when $n<M$ holds, resulting in zero interference. Due to the similarities in behaviour, as already mentioned, the same observations hold for the CE-end scheme but in reverse order, see Figure 5(b).

The CDF curves for the EC-begin ordering policy are shown in Figure 5(c). Immediately we notice that the CDF curves of RBs towards the end of the spectrum are characterised with decreasing probability of high inter-cell interference levels and increasing probability of zero interference. We reason that cell 


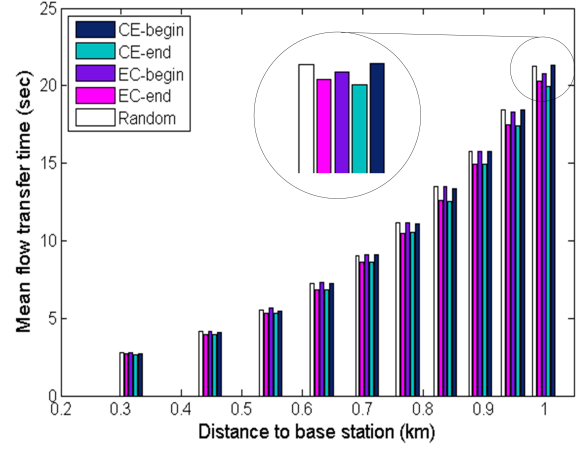

(a) Low load

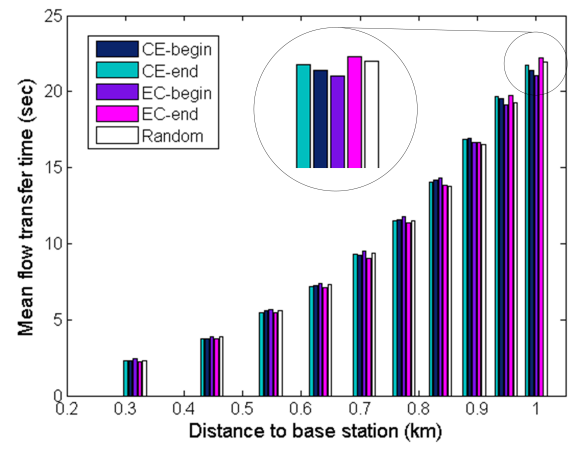

(b) High load

Fig. 6. Mean flow transfer times for lightly (arrival rate $\lambda=0.7$ flows/sec) and heavily (arrival rate $\lambda=4$ flows/sec) loaded system.

edge users (with potentially high $I_{o c}$ ) are served first at the beginning of the spectrum, e.g. at RB1. Subsequent resource blocks are assigned to users located steadily closer to the cell centre, which have (on the average) lower induced interference levels. Finally, RBs at the end of the spectrum, e.g. RB40 and RB50, additionally experience the effects of a non-full system. The observations made for the EC-begin policy also apply for the EC-end policy taking into account the 'reverse' order of RB allocation, see Figure 5(d).

In summary we can say that (i) in a random ordering the inter-cell interference does not depend on the part of spectrum where it exhibits; (ii) a centre to edge ordering results in intersecting CDFs of different RBs, making the final effect on performance difficult to predict; and (iii) policies with edge to centre spatial ordering show potential for interference mitigation. The latter is based on the assumption that neighbour cells apply opposite spectrum allocation, e.g. EC-begin and EC-end, such that the interference experienced by the opposite ends of the spectrum is minimised.

\section{Numerical results - flow-level performance}

In this section we address five evaluation scenarios. In each scenario the $\mathrm{NC}$ adopts one of the five ordering policies (CE-begin, CE-end, EC-begin, EC-end or random) while the $\mathrm{RC}$ always adopts a CE-begin policy; this provides us a common base for comparison. We have evaluated performance in terms of mean flow transfer times for lightly and highly loaded system.

The user performance for each of the evaluation scenarios is presented in Figure 6. Two situations are shown - a lightly (arrival rate $\lambda=0.7$ flows $/ \mathrm{sec}$ ) and a heavily (arrival rate $\lambda=4$ flows/sec) loaded system. In order to ease interpretation of the results, for each situation, we have also shown a zoom in view of the last zone for which the differences in performance are most articulated. 
The general conclusion from Figure 6 is that the particular choice of ordering does not influence significantly the flow-level performance. This may be slightly surprising recalling the big difference in CDF's of the inter-cell interference. We explain this behaviour by the specific choice of scheduling policy, namely a single $\mathrm{RB}$ per user. Under this condition relatively many users (50 with total system bandwidth of $10 \mathrm{MHz}$ ) can be served during one TTI and thus increasing the arrival rate does not lead to drastic changes in performance. We should also account for the fact that under low load the scheme is inefficient in the resource use, i.e. artificially keeps data rates lower than the potentially possible.

Although performance is similar there is a clear change between a lightly and heavily loaded system. In a lightly loaded system ordering from the high end of the spectrum in the NC, e.g. CE-end or EC-end, delivers better results (Figure 6(a)). In a system with low load mainly the RBs close to the spectrum end, where allocation starts, are used; RBs from the middle of the spectrum and further are often not allocated. Hence, starting RB allocation in neighbour cells from the opposite ends of the spectrum leads to zero interference for the majority of RBs in the RC. Starting RB allocation in neighbour cells from the same end of the spectrum results in interfering transmissions. In a highly loaded system however, see Figure 6(b), the CE-begin and EC-begin policies perform better. Under high load the majority of RBs are allocated and inter-cell interference appears in the bigger part of the spectrum. Hence, applying ordering policies in neighbour cells that start from the opposite ends of the spectrum allows to exploit the few interference free RBs occasionally left out due to insufficient users.

We can conclude that (i) the type of ordering policy does not impact flow-level performance significantly, regardless of the system load, and (ii) some performance gains, mainly for low system load, can be achieved if the ordering policies in neighbour cells are carefully chosen. The former behaviour is explained by two factors - with a single RB allocation 50 users can be served in parallel in $10 \mathrm{MHz}$ band and having 50 users in the system (even under high load) is rare. Hence, flow changes only cause minor redistribution in the inter-cell interference over the spectrum, suggesting mostly constant interference levels in the long run.

\section{Conclusions}

This paper discussed the mutual influence scheduling and inter-cell interference have on each other. On the one side, we showed that the inter-cell interference pattern depends on the particular service policy used by the scheduler. On the other side, the study examined the impact of the inter-cell interference on user performance at flow level. The investigations were done for an access-fair scheduling policy with five different approaches of service allocation. Flow-level evaluation was enabled by the use of a hybrid analytical/simulation approach

which benefits from providing insights on the performance while supporting quick evaluation. 
The results show that the type of the ordering policy significantly affects the inter-cell interference pattern but that this scheduler-specific pattern has little impact on the flow-level performance. By including flow-level behaviour in the analysis we were able to show that certain factors that exhibit strongly at packet level do not affect the eventual performance on a longer time scale (at flow level). This conclusion should be drawn with care since it holds for the particularly studied scheduling scheme. Other schemes may show other behaviour. Still, knowledge on scheduler-dependent inter-cell interference can be used when studying scheduling schemes with interference mitigation.

\section{References}

1. 3GPP TS 36.942. LTE; Evolved Universal Terrestrial Radio Access (E-UTRA); Radio Frequency (RF) system scenarios, 2010.

2. T. Bonald and N. Hegde. Capacity gains of some frequency reuse schemes in OFDMA networks. In Proc. of Globecom 2009, Honolulu, Hawaii, USA, pages $1-6,2009$.

3. D. C. Dimitrova. Analysing uplink scheduling in mobile networks. A flow-level perspective. $\mathrm{PhD}$ thesis, Wöhrmann Print Service, 2010.

4. D. C. Dimitrova, H. van den Berg, and G. Heijenk. Scheduler dependent modelling of inter-cell interference in UMTS EUL. In Proc. of NGMAST 2008, Cardiff, UK, 2009.

5. G. Fodor and M. Telek. Performance analysis of the uplink of a CDMA cell supporting elastic services. In Proc. of Networking 2005, Waterloo, Canada, 2005.

6. H. Holma and A. Toskala. LTE for UMTS: OFDMA and SC-FDMA based radio access. John Wiley \& Sons Ltd, 2009.

7. X. Mao, A. Maaref, and K. H. Teo. Adaptive soft frequency reuse for inter-cell interference coordination in SC-FDMA based 3GPP LTE uplinks. In Proc. of Globecom 2008, New Orleans, Louisiana, USA, pages 1-6, 2008.

8. A. Racz, N. Reider, and G. Fodor. On the impact of inter-cell interference in LTE. In Proc. of Globecom 2008, New Orleans, USA, pages 1 -6, nov. 2008.

9. X. Xiang, F. Liu, and Y. Ji. Simulation based performance evaluation of ICI mitigation schemes for broadband wireless access networks. In Proc. of CNS 2008, Montreal, Canada, pages 181-187, 2008.

10. F. Xiangning, C. Si, and Z Xiaodong. An inter-cell interference coordination technique based on users' ratio and multi-level frequency allocations. In Proc. of WiCom 2007, Shanghai, China, pages 799-802, 2007.

11. Z. Xie and B. Walke. Resource allocation and reuse for inter-cell interference mitigation in OFDMA based communication networks. In Proc. of WICON 2010, Singapore-city, Singapore, pages 1-6, 2010.

12. T. Zhang, Z. Zeng, Ch. Feng, J. Cheng, and L. Song. Uplink power allocation for interference coordination in multi-cell OFDM systems. In Proc. of ChinaCom 2008., pages 716-720, 2008. 